\title{
Caracterização da Formação Inicial e da Atuação Profissional de Professores em Escolas Públicas de Ensino Médio
}

\author{
Caracterización de la Formación Inicial y de la Actuación Profesional de \\ Profesores en Escuelas Públicas de Enseñanza Media
}

Description of training initial and the professional performance in

teachers in public schools high school

\author{
Kauana Martins Bonfada) ${ }^{1}$ \\ Ms. Liane Nair Much ${ }^{2}$ \\ Dr. Eduardo Adolfo Terrazzan ${ }^{3}$
}

\begin{abstract}
Resumo
Este trabalho apresenta um estudo referente a uma das ações desenvolvida no âmbito do Projeto de Pesquisa (Guarda-Chuva) intitulado "Necessidades para o Trabalho Docente em Escolas Públicas para a Permanência Efetiva e a Aprendizagem Qualificada dos Alunos de Ensino Médio no Brasil", em desenvolvimento pelo Grupo de Estudos, Pesquisa e Intervenções "Inovação Educacional, Práticas Educativas e Formação de Professores" (INOVAEDUC), sediado no Núcleo de Estudos em Educação, Ciência e Cultura (NEC), do Centro de Educação (CE), da Universidade Federal de Santa Maria (UFSM). Atualmente, são necessárias diversas e variadas ações para uma efetiva qualificação do ensino praticado nas Escolas de Educação Básica. Além disso, várias pesquisas têm tomado como seus objetos de estudo, tanto a Escola como instituição, como a própria organização do Trabalho Escolar. O que se pretende, de modo geral, é contribuir para o desenvolvimento de ações para promoção de mudanças efetivas nessas escolas, o que exige, para tanto, uma compreensão adequada e profunda das formas de organização do Trabalho Escolar. Assim, temos como objetivo caracterizar formação inicial e atuação profissional e a atuação profissional dos professores de Ensino Médio das Escolas Públicas da cidade de Santa Maria/RS.
\end{abstract}

Palavras-Chave: Atuação Profissional; Ensino Médio; Formação Inicial; Trabalho Docente

\section{Resumen}

Este trabajo presenta un estudio sobre una de las acciones desarrolladas en el marco del Proyecto de Investigación (paraguas) intitulado "Necesidades para el trabajo docente en las Escuelas Públicas para la permanencia efectiva y el Aprendizaje Cualificado de los alumnos de Educación Secundaria en Brasil”, en desarrollo por el Grupo de Estudios, Investigación e Intervenciones "Innovación Educativa, Prácticas Educativas y formación de profesores" (INOVAEDUC), con sede en el Núcleo de Estudios en Educación,

\footnotetext{
${ }^{1}$ Aluna do Curso de Mestrado do Programa de Pós-Graduação em Educação (PPGE), do Centro de Educação (CE), da Universidade Federal de Santa Maria (UFSM); Licenciada em Letras Habilitação Língua Portuguesa e suas Respetivas Literaturas, pela UFSM; Santa Maria, Rio Grande do Sul, Brasil; E-mail: kauanabonfada@hotmail.com

${ }^{2}$ Aluna do Curso de Doutorado do Programa de Pós-Graduação em Educação (PPGE), do Centro de Educação (CE), da Universidade Federal de Santa Maria (UFSM); Mestre em Educação pela UFSM; Licenciada em Geografia pela Universidade da Região da Campanha (URCAMP); Professora da Rede Pública Estadual na Cidade de Santa Maria/RS; Santa Maria, Rio Grande do Sul, Brasil; E-mail: lianemuch@ gmail.com

${ }^{3}$ Professor Titular da Universidade Federal de Santa Maria (UFSM); Doutor em Educação pela Universidade de São Paulo (USP); Bolsista de Produtividade em Pesquisa do CNPq; Coordenador do Grupo de Pesquisas INOVAEDUC/UFSM; Santa Maria, Rio Grande do Sul, Brasil; E-mail: terraedu@yahoo.com.br
} 
Ciencia y Cultura (NEC), del Centro de Educación (CE), de lá Universidad Federal de Santa Maria (UFSM). Actualmente, son necessárias diversas y variadas acciones para una efectiva cualificación de la enseñanza practicada en las Escuelas de Educación Básica. Además, varias investigaciones han tomado como sus objetos de estúdio, tanto la Escuela como la institución, como la propia organización del trabajo escolar. Lo que se pretende, de modo general, es contribuir para el desarrollo de acciones para la promoción de cambios efectivas en estas escuelas, lo que exige, para tanto, una comprensión adecuada y profunda de las formas de organización del Trabajo Escolar. Así, tenemos como objetivo caracterizar la formación inicial y la actuación profesional de los profesores de Enseñanza Media de las Escuelas Públicas de la ciudad de Santa Maria/RS.

Palabras claves: Actuación Professional; Enseñanza Media; Formación Inicial; Trabajo Docente.

\begin{abstract}
This article presents a study of one of the developed actions in the context of the main project named "Necessities of the Docent's Work in Brazilian Publics High Schools Student's Effective Stay e Qualified Learning" ("Necessidades para o Trabalho Docente em Escolas Públicas para a Permanência Efetiva e a Aprendizagem Qualificada dos Alunos de Ensino Médio no Brasil”) advanced by the Study Group, Research and Interventions "Inovação Educacional, Práticas Educativas e Formação de Professores" (INOVAEDUC), placed in the Núcleo de Estudos em Educação, Ciência e Cultura (NEC) of the Centro de Educação (CE) from the Federal University of Santa Maria (UFSM). Currently there are need multiples and diverses actions to an effective qualification of the Basic School's teaching act. Furthermore, many researches have been taking it as their study object, both the School as an institution and the School work's organization by it self. Considering this, this article aims to detail the basic formative and professional acting of professors from the Public High Schools placed in the city of Santa Maria/RS.
\end{abstract}

Keywords: Professional Performance; High School; Initial Formation; Teaching Work.

\title{
1. Introdução
}

O Ensino Médio, definido como etapa final da Educação Básica de acordo o Artigo 35 da Lei de Diretrizes e Bases (LDB), tem a duração mínima de 3 anos, e pode estar organizado de duas formas, a saber: (1) formação geral, propedêutica, de continuidade nos estudos, em que predomina como modelo na educação média brasileira, e tem por finalidade elevar o aluno a um nível mais avançado de aprendizagem, ou seja, um ensino preparatório para o nível superior; (2) formação profissional, isto é, de preparação do jovem para o mercado do trabalho.

Dentre as mudanças que vêm ocorrendo para uma futura melhoria no Ensino Médio brasileiro estão atualmente em vigor políticas públicas e programas educacionais. Por esse motivo, o Ensino Médio tem recebido grande atenção por parte do Ministério da Educação, que articulado com a sociedade, vem realizando um grande esforço no sentido de melhorar sua qualidade. Atualmente essas mudanças decorrem, por meio da implementação de políticas públicas e programas educacionais que visam o desenvolvimento de um novo Ensino Médio, por exemplo, o Artigo 35-A da LDB afirma que a Base Nacional Comum Curricular definirá os direitos e os objetivos da aprendizagem do Ensino Médio, conforme as Diretrizes do Conselho Nacional de Educação por meio de uma divisão em áreas do conhecimento e/ou curricular, a saber: (1) linguagens; (2) matemática; (3) ciências da natureza; (4) ciências 
humanas. Nessa perspectiva de trabalho com áreas do conhecimento e/ou curricular, que devemos pensar e refletir sobre a organização e o desenvolvimento do trabalho docente no âmbito do Ensino Médio.

Assim, sabemos que para o desenvolvimento do trabalho docente, o professor precisa estar sempre se atualizando e adquirindo conhecimento, isto é, sabemos que a formação docente não se encerra ao final da graduação, muito pelo contrário, a conclusão de um curso de licenciatura é a etapa inicial da formação, e essa se estende ao longo da vida profissional de cada professor. Defendemos que a formação docente deve ser permanente, pois a sociedade está em constante mudança/modificação, e estes profissionais devem e têm o dever de se atualizarem, ou seja, procurando formas e/ou recursos de estudos, para oferecer uma educação de qualidade aos alunos, que venha ao encontro dos anseios e das necessidades que a sociedade impõe.

Com o intuito de aprofundar seus conhecimentos e enriquecer sua prática pedagógica, muitos professores participam de seminários, cursos, palestras e oficinas. Também, aprendem muito com colegas da própria escola, ou seja, por meio de trocas de materiais, ideias e experiências. No entanto, uma das mais importantes formas de aprendizagens e mudança de atitudes profissional é a reflexão sobre a própria prática. Por meio dela, o professor tem a oportunidade de analisar suas ações, melhorá-las, ou até mesmo modificá-las. Infelizmente essa reflexão crítica sobre o próprio trabalho não é uma prática habitual para muitos professores. Para tanto, refletimos que para que a formação profissional seja completa, é necessário que os professores se assumam como protagonistas de suas formações. Os professores têm de se tornar produtores da sua profissão (NÓVOA, 1995).

A formação inicial de professores possibilita a constituição de uma coletânea de saberes a serem ensinados. Entretanto, é indispensável, mas não o suficiente. Para isso, precisamos considerar todos os aspectos e especificidades da educação escolar, que possui como característica um processo de constituição de saberes. Assim, para que se consiga realizar essa ação com os professores, com consciência e autonomia do seu significado e implicações, é preciso que na formação inicial o professora seja capacitado a ensinar os alunos, e que eles consigam aprender, isto é, realizando o processo de mediação didática entre conhecimento científico e saberes docentes. (LOPES, 1999).

Logo, o professor não pode cair na rotina e ficar apenas com a sua formação inicial, ou seja, parar no tempo, ele precisa ao longo de sua atuação como docente construir os seus saberes, desenvolver sua prática e suas habilidades, e estipular maneiras de como exercer a 
sua profissão. O professor precisa de tempo em tempo se atualizando, adquirindo conhecimento para poder fazer da melhor forma possível o seu trabalho.

No campo profissional, os professores e seu desempenho profissional vêm sendo fortemente responsabilizados por uma fatia considerável dos baixos índices de escolaridade da realidade brasileira. Estudos dedicados à compreensão da educação têm apontado à necessidade da inserção de ações, políticas e propostas que redundem na melhoria da formação e da atuação desses profissionais. Essa constatação coloca o trabalho docente como foco imperativo de pesquisas que busquem contribuir com as propostas e ou parâmetros para a melhoria educacional. (GAMA, TERRAZZAN, 2015, p.11).

\section{Da Formação Inicial até a Atuação Profissional de Professores}

A forma como os futuros profissionais da área da educação são formados impacta no projeto educacional de qualquer nação, com as mudanças que vem ocorrendo nas formas de aprender e ensinar, os cursos de licenciatura devem preparar os futuros professores para dialogarem com a realidade da sala de aula, isto é, para atuarem como mediadores da aprendizagem. A temática "Formação de Professores" é um assunto tratado não somente pelo domínio da Educação, mas pela própria sociedade de modo geral, esse tema é discutido por meio de documentos, por exemplo, leis, diretrizes, normativas legais, trabalhos acadêmicocientíficos, e também, por pesquisadores da área.

Falar e pensar sobre Educação, mais específico sobre Formação de Professores nos dias de hoje, exige uma consideração de paradigmas do campo educacional e da atuação docente no que tange a ciência e o fazer educação. Logo, a formação docente situada no contexto de grandes mudanças no espaço escolar, nas políticas públicas e em teorias e perspectivas, que correspondem às novas demandas e saberes docentes.

De acordo García (1999), quando falamos em formação de professores precisamos assumir determinadas posições sejam elas epistemológicas, ideológicas, históricas, culturais e sociais, pois estas posições situam interseções relativas ao ensino, aos próprios professores e aos alunos, de qualquer nível educacional. Assim, por meio das tendências e rumos sobre a formação de professores o autor destaca:

A Formação de professores é a área de conhecimentos, investigações e de propostas teóricas e práticas que, no âmbito da Didática e da Organização Escolar, estuda os processos através dos quais os professores - em formação ou em exercício - se implicam individualmente ou em equipa, em experiências de aprendizagem através das quais adquirem ou melhoram os seus conhecimentos, competências e disposições, e que lhes permite intervir profissionalmente no desenvolvimento do seu ensino, do currículo e da escola, com o objetivo de melhorar a qualidade da educação que os alunos recebem (GARCÍA, 1999, p. 26). 
Mediante as ideias do autor refletimos que a formação de professores implica em entender a aprendizagem como um processo em andamento, que precisa ter uma análise cuidadosa ao longo de suas etapas e/ou evolução e/ou concretização, para reorganizar conceitos alicerçados na busca da compreensão de novas ideias e valores referente ao ensino. Discutir, pesquisar e pensar sobre a formação de professores é fundamental para um delineamento de novos caminhos para a prática docente, pois o estudo do trabalho do professor no dia a dia como ser histórico e social, pode auxiliar e beneficiar uma nova ordem pedagógica no que se refere à prática docente e formação inicial e/ou continuada.

No que se refere à formação inicial de professores, Garcia (1999) discute que é uma função que vem progressivamente ao longo da história sendo realizada por instituições específicas, e por pessoas especializadas, por meio de currículos, que estabelecem uma sequência, com conteúdos específicos de programas de formação. A formação inicial de professores apresenta três funções, a saber: (1) formação e treino de professores em formação, tendo em vista uma preparação consonante com as funções profissionais que o profissional deverá desempenhar; (2) a instituição formativa tem a função de controle da certificação e/ou a permissão para poder desempenhar o exercício da docência; (3) a instituição promotora da formação dos profissionais da educação tem a dupla função, isto é, promover mudanças no sistema educativo, e também, contribuir para a socialização e reprodução da cultura dominante.

No entanto, no país em que vivemos, Brasil, é baixo o percentual de docentes com formação inicial especifica no componente curricular em que lecionam no Ensino Médio. O percentual de casos críticos de professores com "irregularidades", isto é, atuando em disciplinas especificas não relacionadas à sua formação inicial ocorre na área curricular das Ciências Humanas - Componente Curricular - Sociologia, segundo o Censo Escolar de 2017 (Brasil, 2017).

O professor tem obrigação e compromisso com a comunidade escolar de expor uma competência profissional coerente. O sistema de ensino necessita de um domínio de habilidades, técnicas e recursos para o desenvolvimento de ações didáticas de professores. Entretanto, pela falta de comprometimento de governantes e o desleixo com a Educação no país, o profissional da docência não consegue firmar um compromisso coerente com seus alunos, tendo em vista que atuar em componentes curriculares diferentes de sua formação inicial. 


\section{Procedimentos Metodológicos}

Para o desenvolvimento desse estudo selecionamos 26 Escolas de Ensino Médio da Cidade de Santa Maria/RS. Após A definição das escolas, contatamos todas, para uma visita, de modo a realizar um mapeamento dos professores nelas atuantes, em seguida contatamos a $8^{\mathrm{a}}$ Coordenadoria Regional de Educação ( $8^{\mathrm{a}} \mathrm{CRE}$ ) para auxiliar na coleta de informações complementares necessárias à elaboração de um quadro-síntese resultante desse mapeamento. Além disso, elaboramos os seguintes instrumentos para auxiliar na pesquisa, a saber: (1) um cadastro geral, contendo dados dessas escolas e das equipes diretivas; (2) um quadro-síntese com informações de atuação dos professores, sinalizando, para cada um deles, os componentes curriculares sob suas responsabilidades, modalidade de ensino em que atuam, suas cargas horárias e seus regimes de trabalho; (3) um quadro-síntese com informações referentes à formação acadêmica de cada professor.

Entretanto, para este trabalho realizamos um recorte, ou seja, escolhemos apenas 8 escolas, por meio do seguinte critério de coleta de informações para a pesquisa: divisão das escolas, por regiões administrativas estabelecidas pela Prefeitura Municipal da Santa Maria/RS: (1) centro-urbano; (2) norte; (3) nordeste; (4) leste; (5) centro-leste; (6) sul; (7) centro-oeste; (8) oeste.

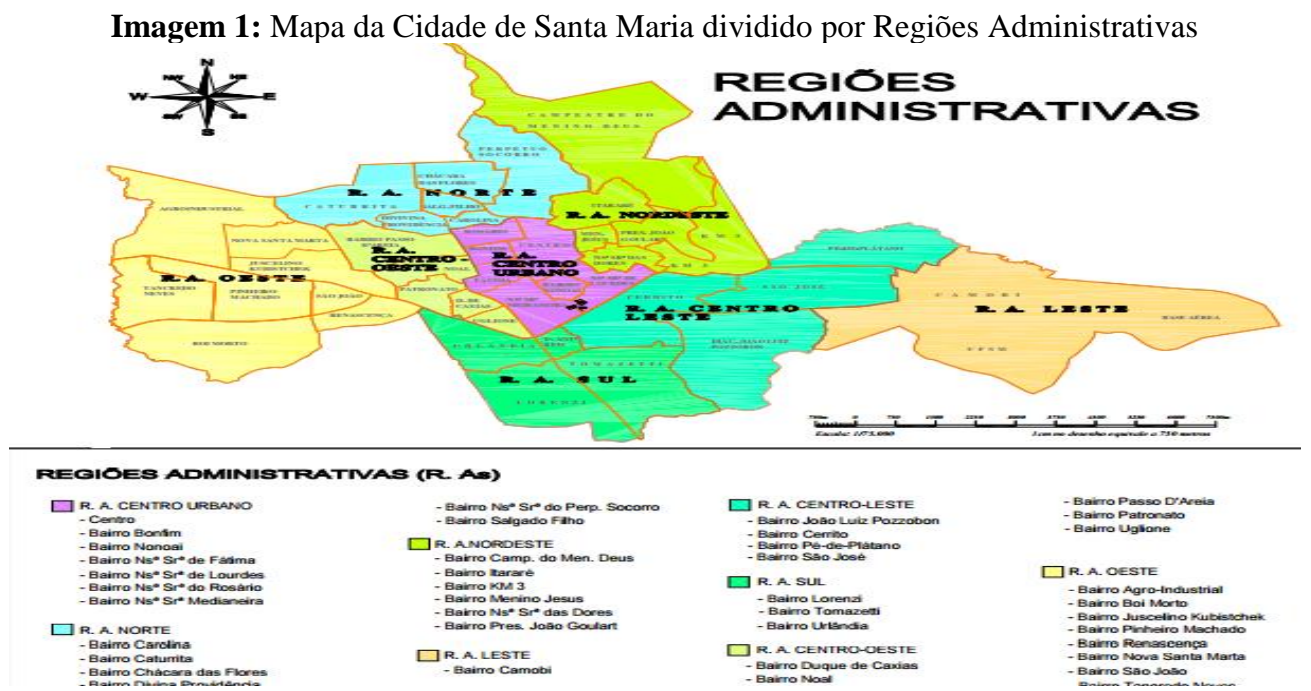

Fonte: Site da Prefeitura Municipal de Santa Maria/RS. Disponível em: <http://www.santamaria.rs.gov.br/index.php>

Para a organização e o tratamento das informações coletadas, fazemos uso da Teoria Fundamentada como um guia para desenvolver a pesquisa. $\mathrm{O}$ pesquisador pode fazer o uso dessa teoria tanto em pesquisas de natureza quantitativa quanto qualitativa, em que o intuito seja de analisar os dados de modo a procurar entender determinadas situações, por exemplo, como e porque os participantes da pesquisa agem de determinada maneira, já que se parte dos 
RELACult - Revista Latino-Americana de Estudos em Cultura e Sociedade

dados para chegar a uma teoria sobre o fenômeno analisado. Para um melhor entendimento do estudo, organizamos os dados no quadro abaixo:

Tabela 01: Quantitativo de docentes por escolas

\begin{tabular}{|c||c|c|c|c|c|c|c|c||}
\hline \multirow{2}{*}{ QUANTIDADE } & \multicolumn{7}{|c|}{ ESCOLAS DE ENSINO MÉDIO SELECIONADAS } \\
\cline { 2 - 11 } & E01 & $\mathbf{E 0 2}$ & $\mathbf{E 0 3}$ & $\mathbf{E 0 4}$ & $\mathbf{E 0 5}$ & $\mathbf{E 0 6}$ & $\mathbf{E 0 7}$ & E08 \\
\hline Núm. Total de Professores & 18 & 23 & 56 & 18 & 11 & 11 & 18 & 31 \\
\hline Área das Humanas & 07 & 10 & 22 & 06 & 05 & 05 & 08 & 15 \\
\hline Área de Ciências da Natureza & 06 & 07 & 15 & 05 & 03 & 03 & 02 & 07 \\
\hline Área das Linguagens & 09 & 12 & 23 & 11 & 06 & 05 & 09 & 15 \\
\hline Área da Matemática & 02 & 02 & 06 & 02 & 01 & 02 & 02 & 02 \\
\hline \hline
\end{tabular}

Fonte: produzido pelos autores

\section{Discussão e Constituição dos Resultados}

A partir do quadro apresentado anteriormente podemos perceber que ocorre uma oscilação entre os dados. Para constituir a nossa amostra escolhemos 8 escolas da cidade de Santa Maria. Dentre essas escolas, todas apresentarem "irregularidades" nas condições de trabalho desses docentes. O quadro abaixo é apenas uma imagem ilustrativa para um melhor entendimento dos dados coletados.

Tabela 02: Quantitativo de docentes por área curricular

\begin{tabular}{|c|c|c|c|c|c|c|}
\hline \multicolumn{7}{|c|}{ "DESCRIÇÃO DAS ESCOLAS } \\
\hline \multirow{2}{*}{ Sigla } & $\begin{array}{c}\text { Localização } \\
\text { Santa Maria/RS }\end{array}$ & \multicolumn{5}{|c|}{ Quantidade de Professores } \\
\hline & Regiões & Total & $\begin{array}{c}\text { Área } \\
\text { Ciên. Nat. }\end{array}$ & $\begin{array}{c}\text { Área } \\
\text { Ciên. Hum }\end{array}$ & $\begin{array}{l}\text { Área } \\
\text { Ling. }\end{array}$ & $\begin{array}{l}\text { Área } \\
\text { Mat. }\end{array}$ \\
\hline E01 & Nordeste & 18 & $\begin{array}{l}\bullet \mathrm{F}=02 \\
\bullet \mathrm{Q}=02 \\
\bullet \mathrm{B}=02\end{array}$ & $\begin{array}{l}\bullet \mathrm{G}=02 \\
-\mathrm{H}=02 \\
\cdot \mathrm{S}=01 \\
-\mathrm{F}=01 \\
-\mathrm{ER}=01\end{array}$ & $\begin{array}{l}-\mathrm{A}=01 \\
-\mathrm{LP}=02 \\
-\mathrm{Lit}=02 \\
-\mathrm{LE}=01 \\
-\mathrm{LI}=02 \\
-\mathrm{EF}=01\end{array}$ & - $\mathrm{M}=02$ \\
\hline E02 & Leste & 23 & $\begin{array}{l}\bullet \mathrm{F}=02 \\
-\mathrm{Q}=03 \\
\cdot \mathrm{B}=02\end{array}$ & $\begin{array}{l}-\mathrm{G}=02 \\
-\mathrm{H}=01 \\
-\mathrm{S}=03 \\
-\mathrm{F}=02 \\
-\mathrm{ER}=02\end{array}$ & $\begin{array}{l}-\mathrm{A}=03 \\
-\mathrm{LP}=01 \\
-\mathrm{Lit}=02 \\
-\mathrm{LE}=01 \\
-\mathrm{LI}=03 \\
-\mathrm{EF}=02\end{array}$ & - $\mathrm{M}=02$ \\
\hline E03 & Centro-Urbano & 56 & $\begin{array}{l}-\mathrm{F}=05 \\
-\mathrm{Q}=06 \\
\cdot \mathrm{B}=04\end{array}$ & $\begin{array}{l}-\mathrm{G}=05 \\
\bullet \mathrm{H}=06 \\
\bullet \mathrm{S}=04 \\
\bullet \mathrm{F}=04 \\
-\mathrm{ER}=03\end{array}$ & $\begin{array}{l}-\mathrm{A}=03 \\
-\mathrm{LP}=07 \\
-\mathrm{Lit}=05 \\
-\mathrm{LE}=02 \\
-\mathrm{LI}=03 \\
-\mathrm{EF}=03\end{array}$ & - $\mathrm{M}=06$ \\
\hline
\end{tabular}


RELACult - Revista Latino-Americana de Estudos em Cultura e Sociedade

Revista Latinoamericana de Estudios en Cultura y Sociedad | Latin American Journal of Studies in Culture and Society V. 04, ed. especial, fev., 2018, artigo n ${ }^{\circ} 797$ | relacult.claec.org | e-ISSN: 2525-7870

\begin{tabular}{|c|c|c|c|c|c|c|}
\hline \multicolumn{7}{|c|}{ DESCRIÇÃO DAS ESCOLAS } \\
\hline \multirow{2}{*}{ Sigla } & $\begin{array}{c}\text { Localização } \\
\text { Santa Maria/RS }\end{array}$ & \multicolumn{5}{|c|}{ Quantidade de Professores } \\
\hline & Regiões & Total & $\begin{array}{c}\text { Área } \\
\text { Ciên. Nat. }\end{array}$ & $\begin{array}{c}\text { Área } \\
\text { Ciên. Hum } \\
\end{array}$ & $\begin{array}{l}\text { Área } \\
\text { Ling. }\end{array}$ & $\begin{array}{l}\text { Área } \\
\text { Mat. }\end{array}$ \\
\hline E04 & Norte & 18 & $\begin{array}{l}\bullet \mathrm{F}=02 \\
\bullet \mathrm{Q}=01 \\
\bullet \mathrm{B}=02\end{array}$ & $\begin{array}{l}\bullet \mathrm{G}=01 \\
\bullet=02 \mathrm{H} \\
\bullet \mathrm{S}=01 \\
\bullet \mathrm{F}=01 \\
\bullet \mathrm{ER}=01\end{array}$ & $\begin{array}{l}\mathrm{A}=01 \\
-\mathrm{LP}=03 \\
-\mathrm{Lit}=02 \\
-\mathrm{LE}=01 \\
-\mathrm{LI}=02 \\
-\mathrm{EF}=02\end{array}$ & - $\mathrm{M}=02$ \\
\hline E05 & Oeste & 11 & $\begin{array}{l}-\mathrm{F}=01 \\
-\mathrm{Q}=01 \\
-\mathrm{B}=01\end{array}$ & $\begin{array}{l}\bullet \mathrm{G}=01 \\
\bullet \mathrm{H}=01 \\
\bullet \mathrm{S}=01 \\
-\mathrm{F}=01 \\
-\mathrm{ER}=01\end{array}$ & $\begin{array}{l}-\mathrm{A}=01 \\
-\mathrm{LP}=01 \\
-\mathrm{Lit}=01 \\
-\mathrm{LE}=01 \\
-\mathrm{LI}=01 \\
-\mathrm{EF}=01\end{array}$ & $\bullet \mathrm{M}=01$ \\
\hline E06 & Centro-Leste & 11 & $\begin{array}{l}\bullet \mathrm{F}=01 \\
\bullet \mathrm{Q}=01 \\
\bullet \mathrm{B}=01\end{array}$ & $\begin{array}{l}\bullet \mathrm{G}=01 \\
\bullet \mathrm{H}=01 \\
\bullet \mathrm{S}=01 \\
\bullet \mathrm{F}=01 \\
\bullet \mathrm{ER}=01\end{array}$ & $\begin{array}{l}-\mathrm{A}=02 \\
-\mathrm{LP}=? ? ? \\
-\mathrm{Lit}=01 \\
-\mathrm{LE}=? ? ? \\
-\mathrm{LI}=01 \\
-\mathrm{EF}=01\end{array}$ & - $\mathrm{M}=02$ \\
\hline E07 & Sul & 31 & $\begin{array}{l}-\mathrm{F}=02 \\
-\mathrm{Q}=03 \\
-\mathrm{B}=02\end{array}$ & $\begin{array}{l}\bullet \mathrm{G}=03 \\
\bullet \mathrm{H}=03 \\
-\mathrm{S}=03 \\
-\mathrm{F}=03 \\
-\mathrm{ER}=03\end{array}$ & $\begin{array}{l}-\mathrm{A}=02 \\
-\mathrm{LP}=03 \\
-\mathrm{Lit}=03 \\
-\mathrm{LE}=03 \\
-\mathrm{LI}=02 \\
-\mathrm{EF}=02\end{array}$ & - $\mathrm{M}=02$ \\
\hline E08 & Centro-Oeste & 18 & $\begin{array}{l}-\mathrm{F}=00 \\
-\mathrm{Q}=01 \\
-\mathrm{B}=01\end{array}$ & $\begin{array}{l}\bullet \mathrm{G}=01 \\
\bullet \mathrm{H}=01 \\
\bullet \mathrm{S}=03 \\
\bullet \mathrm{F}=02 \\
-\mathrm{ER}=01\end{array}$ & $\begin{array}{l}-\mathrm{A}=02 \\
-\mathrm{LP}=02 \\
\cdot \mathrm{Lit}=02 \\
\cdot \mathrm{LE}=02 \\
\cdot \mathrm{LI}=01 \\
\cdot \mathrm{EF}=00\end{array}$ & - $\mathrm{M}=02$ \\
\hline
\end{tabular}

Fonte: produzido pelos autores

Para compreendermos melhor essa "irregularidade" nas condições de trabalho dos docentes. Exemplificamos os dados na seguinte forma:

Tabela 03: Quantitativo de casos irregulares

\begin{tabular}{||c|c|c||}
\hline ESCOLAS & TOTAL DE PROFESSORES & CASOS IRREGULARES \\
\hline \hline E01 & 18 & 08 \\
\hline E02 & 23 & 10 \\
\hline E03 & 56 & 05 \\
\hline E04 & 18 & 05 \\
\hline E05 & 11 & 04 \\
\hline E06 & 11 & 07 \\
\hline E07 & 31 & 07 \\
\hline E08 & 18 & 072 \\
\hline
\end{tabular}

Fonte: produzido pelos autores 
O que seriam essas "irregularidades". Em algumas escolas existem profissionais sem formação alguma para atuarem na área da docência, por exemplo, bacharéis em diversas áreas. Ou até mesmo professores não habilidades para alguns componentes curriculares, trabalhando para poder cumprir a carga horária, ou até mesmo porque a secretaria do estado não disponibilizou professor para determinada disciplina. Entre os casos mais comuns, são: professores de geografia e/ou história ministrando aulas de filosofia, sociologia ou ensino religioso, como também, professores de língua portuguesa sem habilitação alguma para língua estrangeira, ministrando disciplinas de inglês e espanhol. Como mencionado anteriormente na área das Ciências Humanas, mais especifico no componente curricular de Sociologia, de acordo o Censo Escolar de 2017 (Brasil, 2017) é o que apresenta o maior número de casos de irregularidades.

\section{Conclusões}

A escola tem papel fundamental na construção do conhecimento e na consolidação de aprendizagens. Para esse processo pedagógico ser significativo é importante que seus docentes adotem práticas de ensino diferenciadas e condizentes com a necessidade contemporânea, que façam a diferença no cotidiano dos alunos. Nesse contexto a escola deve ser vista além do lugar que socializa o saber metódico, mas também como um lugar de trabalho do professor, o qual depende, para se organizar e atender às suas demandas, de profissionais que atuem em seus diferentes espaços, de forma sustentada por conhecimentos e saberem construídos com base em diferentes teorias e experiências diversificadas. Mas, para isso o professor deve estar capacitado, ou seja, ele deve atuar na sua área de formação, naquela que escolheu para atuar. Assim, é possível perceber uma ênfase na prática como componente curricular dos cursos de formação inicial de professores como oportunidade de refletir sobre o processo inicial de construção da identidade profissional.

\section{Referências}

BRASIL. Ministério da Educação. Inep. Censo escolar da educação básica. Brasília, 2017. Disponível em:

http://download.inep.gov.br/educacao_basica/censo_escolar/notas_estatisticas/2017/notas_est atisticas_censo_escolar_da_educacao_basica_2016.pdf >. Acesso em: 18.dez.2017.

GAMA, M. E. R; TERRAZZAN, E. A. O trabalho docente em uma escola pública de Educação Básica: entre a complexidade e a simplicidade.1.ed. Curitiba, PR: CRV 2015. 
LOPES, A. R. C. Conhecimento escolar. Ciência a cotidiano. Rio de Janeiro, RJ. Editora da UERJ, 1999.

MARCELO, G. C. Formação de professores: para uma mudança educativa. Tradução de Isabel Narciso. Porto/PT: Porto. (Coleção "Ciências da Educação Século XXI", 2), 1999.

NÓVOA, A. Diz-me como ensinas, dir-te-ei quem és e vice-versa. In.: FAZENDA, Ivani. (Org.). A pesquisa em educação e as transformações do conhecimento. Campinas, SP: Papirus, 1995. 